\title{
A New Approach to Automatic Reconstruction of a 3-D World Using Active Stereo Vision
}

\author{
Chung-Yi Lin
}

Institute of Information Science, Academia Sinica, Taipei 115, Taiwan, and Department of Computer Science and Information Engineering, National Taiwan University, Taipei 106, Taiwan

\author{
Sheng-Wen Shih ${ }^{1}$ \\ Department of Computer Science and Information Engineering, National Chi Nan University, \\ Nantou 545, Taiwan \\ E-mail: swshih@ncnu.edu.tw
}

\section{Yi-Ping Hung}

Institute of Information Science, Academia Sinica, Taipei 115, Taiwan

E-mail: hung@iis.sinica.edu.tw

and

\section{Gregory Y. Tang}

Department of Computer Science and Information Engineering, National Taiwan University, Taipei 106, Taiwan

Received March 6, 2000; accepted October 3, 2001

In this paper, we propose a new automatic approach to reconstructing 3-D environments using an active binocular head. To efficiently store and access the depth estimates, we propose the use of an inverse polar octree which can transform both unbounded depth estimates and unbounded estimation errors into a bounded 3-D space with appropriate resolution. The depth estimates are computed by using the asymptotic Bayesian estimation method. Estimated depth values are then smoothed by using discontinuity-preserving Markov random fields. The path of the local motion required by the asymptotic Bayesian method is determined online automatically to reduce the ambiguity of stereo matching. Rules for checking the consistency between the new observation and the previous observations have been developed to properly update the inverse polar octree. Experimental results showed that the proposed approach is very promising for automatic generation of 3-D models which can be used for rendering a 3-D scene in a virtual reality system. (c) 2002 Elsevier Science (USA)

${ }^{1}$ To whom correspondence should be addressed. 
Key Words: active vision; stereo vision; 3-D reconstruction; asymptotic Bayesian estimation; 3-D data integration; view interpolation.

\section{INTRODUCTION}

In the past few years, virtual reality (VR) has found many applications in different areas, such as education, business, and entertainment. Because of the rapid growth of VR applications, automatic generation of 3-D models from images has attracted much attention. To reconstruct 3-D models from images, many people choose to use the stereo vision techniques. However, it is well known that stereo correspondence is a very difficult problem. The results of 3-D reconstruction obtained by using automatic stereo matching algorithms (such as those described in [1]) are still not reliable enough for practical use. Successful 3-D reconstruction systems used at present still must utilize structured light (e.g., laser light stripe) or impose strong constraints (e.g., the continuous surface constraint) to simplify the stereo matching problem. Otherwise, human interaction must be introduced into the reconstruction system to solve the general stereo correspondence problem (e.g., Debevec et al. [2] and Hung et al. [3]). About one decade ago, Alominos and Badyopadhyay showed that many computer vision problems which are ill-posed, nonlinear, and unstable for a passive observer become well-posed, linear, and stable for an active observer [4]. Their work is based on the assumption that the active vision system is well calibrated so that accurate camera parameters are always available with respect to any configuration of the active vision system. However, most of the existing active vision systems are not accurately calibrated for two main reasons: (1) many vision tasks can be accomplished without having to reconstruct the 3-D model of a scene [5], and (2) existing calibration techniques developed for passive vision systems are not suitable for active vision systems. Nevertheless, since a calibrated active vision system has many advantages over an uncalibrated system, people are developing calibration techniques for active vision systems according to their requirements. Reid and Beardsley have developed a method for aligning a pair of stereo cameras so that their optical axes are parallel [6]. Based on a simplified kinematics model, McLauchlan and Murray at Oxford used a variable state-dimension filter to recursively estimate the head/eye orientation relation and the vertical and horizontal effective focal lengths without having to use a special calibration object [7]. The KTH group developed a three-stage calibration method, i.e., zoom lens calibration [8, 9], kinematic calibration [10], and head/eye calibration [11], for their active binocular head. We have also spent many years developing a four-stage calibration method for our binocular head and have achieved very accurate calibration results [12]. Based on our well-calibrated binocular head (referred to as the IIS head), we have the ability to try to solve the 3-D reconstruction problem using the active vision paradigm. Reconstructing the 3-D environment map using an active vision system involves the following three main problems:

1. The where-to-look-next problem: The main advantage of an active vision system over a passive system is that some of the external and internal camera parameters of the active vision system can be adaptively controlled so that an assigned vision task can be accomplished in a more efficient and accurate way. In particular, when a very specific vision task, e.g., human-made object recognition [13], face recognition [14], mobile robot navigation [15], and visual pursuing [16], is assigned to an active vision system, the criteria for selecting 
the best next observation configuration for the system are well defined. On the other hand, if there is no specific goal set for the vision system, then the where-to-look-next problem will become too subjective so that no generally acknowledged criterion can be found. In this work, the given vision task is to reconstruct a textured 3-D environment map that is visually consistent with the observed images. When there is no prior knowledge about the environment, each viewpoint is of equal importance because we need as many as possible images to detect and to remove visually inconsistent 3-D data. Therefore, we can simply choose to use a uniform sampling strategy to acquire images at equally spaced viewpoints. Notice that determining the next best observation configuration for reconstructing a global scene is different from determining the best configuration for reconstructing a small 3-D surface patch because the latter configuration has a more specific goal than the former.

2. Navigation problem: Once the next best viewpoint has been determined, the active vision system should have the ability to safely navigate itself to the viewpoint. However, since robot navigation has been extensively studied for decades (refer to [17]) and it is not the research focus of this work, we will assume that our active vision system is moving in an obstacle-free environment.

3. Integration of the estimated 3-D data problem: As the active vision system moves to a new viewpoint and computes new 3-D estimations, we must update the 3-D map so that it is consistent with the new observations. The main difficulty of data integration is that when the new observations are inconsistent with the old observations, we must determine which observations are incorrect even though both the new and old data are estimated using the same method.

For the VR applications, the virtual 3-D scene can be rendered by computing the shading of an object given the position of light sources and surface properties of the object. In this way, the 3-D model of the object must be very accurate because the computed shading appearance is very sensitive to 3-D noise. On the other hand, when image-based techniques are used to render a 3-D scene with texture extracted from real images, the quality of the rendered image is more tolerable of inaccurate 3-D data. Therefore, with image-based rendering techniques, one does not have to reconstruct a very accurate 3-D model in order to obtain a photo-realistic VR scene [18-20]. Knowing that image-based rendering techniques do not require accurate 3-D reconstruction and that 3-D reconstruction is, in general, an ill-posed problem, we do not intend to reconstruct highly accurate 3-D models of the scene. Instead, our goal is to reconstruct an approximate 3-D model having some associated texture information so that this approximation model, together with the texture information, can be used to synthesize images which look similar to the real images when observed from arbitrary viewpoints within a prespecified viewing area. If the synthesized image looks different from the real image when observed from a new point of view, then our goal is to update the current scene model so that the model will be consistent with all the previous views the vision system had observed. We hope that the scene model will become more accurate, as the vision system samples more viewpoints within the specified viewing area. The idea, which we have proposed independently in [19], of reconstructing a textured 3-D model that is visually consistent with the observed images is similar to the space carving technique proposed by Kutulakos and Seitz [20]. They call the reconstructed approximated 3-D model the photo-consistent shape. In the absence of a priori geometric information, reconstructing a photo-consistent shape of an object is more feasible than reconstructing its exact 3-D model. The main difference between our approach and the space carving technique 
is that our method is based on stereo vision techniques for 3-D estimation. But the space carving technique does not directly compute the 3-D data; instead, it simply removes all non-photo-consistent surface voxels. The advantage of the space carving approach is that the computation algorithm is very simple and relatively robust. But it will need to use many images sampled densely in 3-D space in order to generate a visually compelling photoconsistent shape. On the other hand, if only a handful of calibrated images are available, then we will have to use stereo vision techniques to reconstruct a 3-D model. However, since the computed stereo correspondences usually contain some mismatched pairs, it is then necessary to introduce an error-correction mechanism into the system to update the 3-D data. In our method, the error-correction mechanism is first to detect visually inconsistent 3-D data and then to reestimate them. In our point of view, our method is kind of a combination of the surface estimation technique and the space carving technique. In particular, when one is trying to reconstruct a wide scene, the proposed method can provide a visually consistent 3-D model with fewer images than the space carving technique requires.

In addition to the space carving technique, many approaches based on active vision systems for automatic generation of 3-D models from images have been proposed in the past decades. Beß et al. used a calibrated active color camera for 3-D reconstruction [21]. Three-dimensional data were estimated from monocular color image sequences by using a stereo technique which combines the feature-based and correlation-based stereo matching methods. Disparities obtained from a feature-based stereo were used to guide correlationbased stereo matching. Maru et al. used a stereo rig, which was mounted on a calibrated translation stage so that the orientation of the translation stage with respect to the camera coordinate systems was known; hence, rough depth estimates could be obtained from the detected optical flow and then used to guide stereo matching [22]. Grosso and Tistarelli used a binocular head for robot visual guidance [23]. Since the task of visual guidance does not require accurate 3-D models of the environment, the calibration task can be simplified, as only a few parameters need to be roughly estimated. In their work, a simple method for estimating some necessary camera parameters was proposed and methods for estimating the rough depth value of the environment as well as the time-to-impact were developed. Ahuja and Abbott studied the 3-D reconstruction problem using an active stereo system [24]. To reconstruct the surface from stereo images, they argued that the tightly coupled use of focus, camera vergence, and stereo disparity results in a more powerful and complete system for surface estimation than when those cues are used individually. In their approach, aperture setting, focus setting, vergence angle, depth estimates, and the integration of depth estimates from different cues and different viewpoints can be determined by minimizing a criterion function with a smoothness constraint. In their experiments, object surfaces were covered with textured newspapers to make stereo matching and depth from a focusing visual module provide reliable results. Ours is distinct from theirs in that we have a well-calibrated active binocular head, and we can apply the active sensing paradigm to simplify the 3-D reconstruction problem, to achieve more precise stereo matching results, and to integrate 3-D estimates from multiple viewpoints; hence, we are able to deal with the reconstruction problem of a more complicated environment. Also, instead of applying the active vision system to reconstruct a narrow scene, our goal is to reconstruct a wide scene. Marchand and Chaumette have developed an active vision system consisting of a monocular camera mounting on the end effector of a 6 degrees of freedom Cartesian robot [25]. Their active vision system was accurately calibrated so that $3-\mathrm{D}$ data can be computed using structure 
from controlled motion methods. Unknown static scenes containing objects of simple shapes can be automatically explored and reconstructed using images acquired and processed at nearly video rate. The reconstruction results of simple scenes (several light color objects plus a black background) were very accurate, but their method was developed for polyhedral objects and cylinders only. We do not impose such strong constraints on the scenes in our method; therefore, we do not intend to achieve accuracy comparable to that of their system.

In this paper, we propose a new approach to reconstructing a model of the 3 -D environment automatically by using a well-calibrated active binocular head [12]. The reconstructed 3-D points and their gray level values are stored in a volumetric data structure, i.e., the inverse polar octree (IPO), which will be described in Section 2. An active control scheme has been used to minimize the ambiguity in stereo matching. The 3-D structure of the scene is estimated by using the asymptotic Bayesian estimation method [26], which is similar to the multiple-baseline stereo method [27] except that the asymptotic Bayesian estimation processes only one image at a time and it also provides the uncertainty information of the depth estimates. Details of the reconstruction process is described in Section 3. Some experimental results on reconstructing the 3-D model of complex scenes are presented in Section 4. Conclusions are given in Section 5.

\section{INVERSE POLAR OCTREE}

In this work, we chose to use the voxel-based data structure for storing the reconstructed 3-D data because the overhead of integrating noisy 3-D estimations with a voxel-based data structure is smaller than that with a mesh-based data structure. When the 3-D reconstruction process is completed, the voxel-based 3-D data can be transformed into a mesh-based representation for fast rendering. To use the voxel-based 3-D representation, we must solve the problem of packing the 3-D information contained in the infinite 3-D space into the finite memory space in a computer. To deal with this problem, we found that the 3-D measurement error of a stereo vision system is proportional to the distance between the object and the stereo cameras [28]. This fact suggests that uniform quantization of the 3-D data obtained by the stereo vision system is inefficient. A better quantization scheme is to have the resolution of the volumetric representation inversely proportional to the object distance. However, nonuniform quantization will result in complicated octree representations. Our solution to this problem is to take an inverse polar transformation of the estimated 3-D data before quantizing them into voxels. The inverse polar transformation is described in the following:

1. Transform the 3-D Cartesian coordinates, $(x, y, z)$, of a point $P_{3 D}$ to spherical coordinates, $(\rho, \theta, \phi)$, where $\rho$ is the distance from the origin to point $P_{3 D}$, and $\theta$ and $\phi$ are the angles specifying the direction of a unit vector pointing from origin to point $P_{3 D}$.

2. For the 3-D spherical coordinates, $(\rho, \theta, \phi)$, compute its inverse polar coordinates, $\left(\frac{1}{\rho}, \theta, \phi\right)$.

There are two major advantages to taking the inverse polar transformation. The first advantage is that, after the transformation, all the surrounding 3-D objects farther than a minimum distance to the observer, say $R_{\text {min }}$, will be enclosed within a sphere with radius $\frac{1}{R_{\min }}$. In this way, the infinite $3-\mathrm{D}$ world outside a sphere is now mapped into a finite sphere, as shown in Fig. 1. The second advantage is that, after taking the inverse polar transformation, 


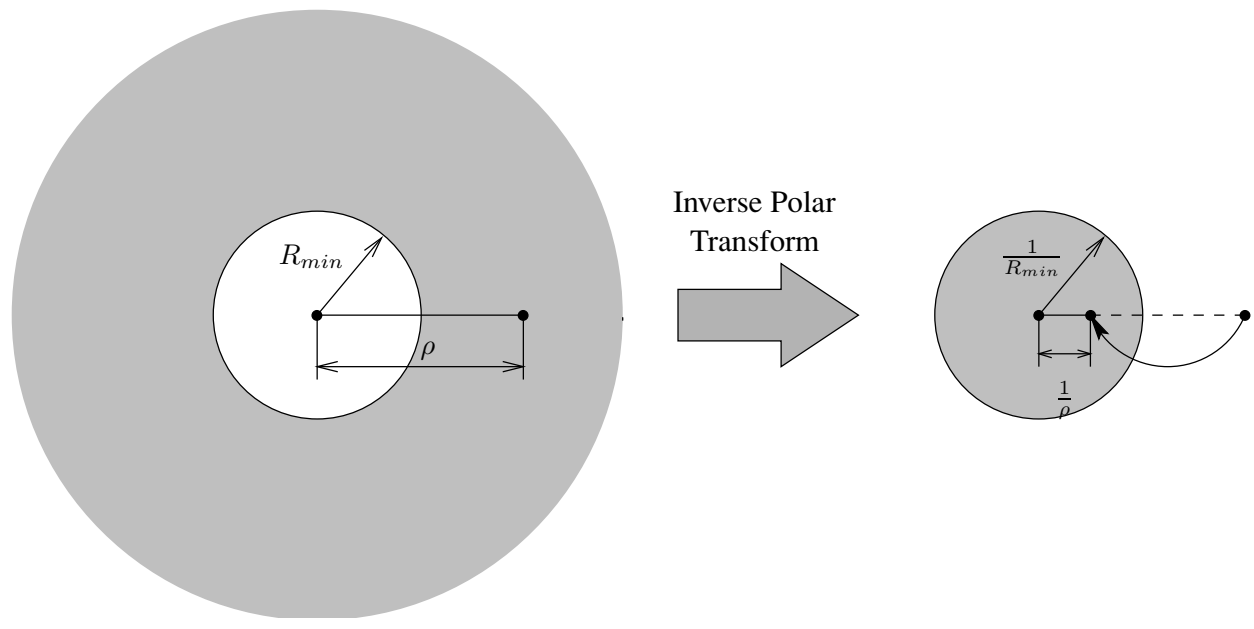

FIG. 1. Inverse polar transform.

we can apply the uniform quantization scheme because the estimation error is now bounded, which is explained below in more detail.

Let $\rho$ be the distance of an object point away from the observer. Since the 3-D estimation error is proportional to the object distance, the 3-D estimation error of the object point is approximately $k \rho$, where $k$ is a constant determined by the configuration of the stereo cameras. Therefore, the estimate of the object distance is approximately $(1+k) \rho$. Notice that the estimation error, $k \rho$, is unbounded because the estimation error will approach infinity as $\rho$ approaches infinity. However, after the inverse polar transformation, the object distance is now mapped to $\frac{1}{\rho(1+k)}$. Since the 3-D estimation error is, in general, much smaller than $\rho$, i.e., $k \ll 1$, we have

$$
\frac{1}{\rho(1+k)} \approx \frac{1}{\rho}-\frac{k}{\rho}
$$

Here, the second term of the right-hand side of (1) is the transformed estimation error, which is now bounded by $\frac{k}{R_{\min }}$, if $\rho>R_{\min }$. If we choose the quantization unit to be $\frac{k}{R_{\min }}$, then the estimation error will be less than the quantization error for all the object points outside the sphere of radius $R_{\min }$. It is advantageous to have a quantization error larger than the estimation error when using volumetric representation in 3-D reconstruction, because if the estimation error is larger than the quantization unit, then there will be many undesired false voxels that are caused by the estimation noise and are located around the real object position. As a result, when the quantization unit is smaller than $\frac{k}{R_{\min }}$, we will not only have to use much larger amounts of memory to store the 3-D data but also will obtain sparser scattering of 3-D measurement data. Sparsely scattered 3-D data will make the data integration more difficult; thus it is unwanted.

After we take the inverse polar transformation, 3-D data are stored in an octree according to the three coordinate components, $\frac{1}{\rho}, \theta$, and $\phi$. Let $\Delta \theta$ and $\Delta \phi$ be the angular resolution of the octree. The octree is created in the spherical coordinate system to maintain the uniform angular resolution, as shown in Fig. 2. Two 3-D points with 3-D coordinates $(\rho, \theta, \phi)$ and $(\rho, \theta+\Delta \theta, \phi+\Delta \phi)$ will be stored at $\left(\frac{1}{\rho}, \theta, \phi\right)$ and $\left(\frac{1}{\rho}, \theta+\Delta \theta, \phi+\Delta \phi\right)$, respectively. 


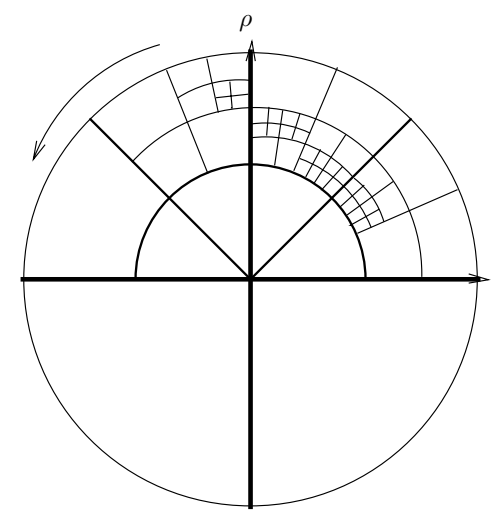

(a)

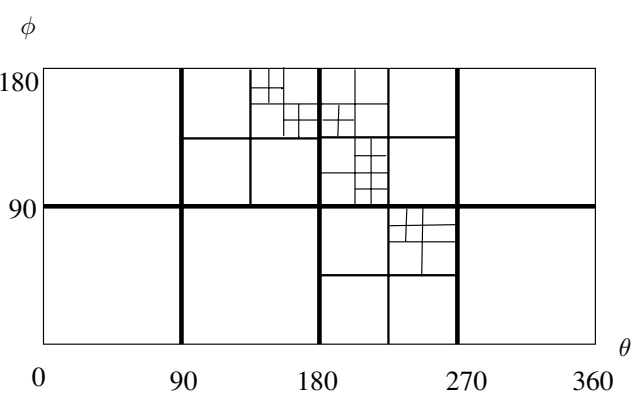

(b)

FIG. 2. An illustration of the inverse polar octree.

Obviously, the angular resolution of the octree remains unchanged after the inverse polar transformation and the uniform quantization into an octree.

\subsection{Some Practical Issues of Using the Inverse Polar Octree}

Notice that the inverse polar transformation is introduced in this work to map an infinite set

$$
\left\{p \in R^{3},\|p\| \geq R_{\min }\right\}
$$

into a finite space

$$
\left\{q \in R^{3},\|q\| \leq \frac{1}{R_{\min }}\right\} .
$$

Thus we can quantize the finite space and store the reconstructed 3-D information in a limited memory space of a computer. However, introducing the inverse polar transformation also brings some problems because it makes the volumetric representation become viewer-centered. For a viewer-centered representation, when the observer moves to a new position, the entire 3-D data would have to be transformed to a new center accordingly. Due to the quantization scheme that we have adopted (the inverse polar octree), 3-D data at different distances to the center contain different levels of quantization error. When the observation center jumps to a new point at a long distance far away from the original center, the nonuniform quantization error may cause the transformation to become more time-consuming because a voxel in the original octree may be mapped to multiple voxels in the other octree and vice versa. However, it is rare that a moving observer would change its viewpoint in this way. In general, the observation center usually moves slowly; thus the levels of quantization error associated with the 3-D data also change slowly and can be approximately regarded as constants. In this case, only simple point-to-point transformations are required in performing the recentering computation. But since the quantization error does not change evidently, an alternative approach is to use an IPO with a fixed center so 
that we can skip the recentering computation. This approach is especially suitable when the prespecified viewing area is small and the IPO is centered at the viewing area.

Notice that our goal is to reconstruct a textured 3-D model that is visually consistent with the images acquired in a prespecified viewing area. Therefore, it is not guaranteed that images synthesized using the reconstructed model are still serviceable if the specified points of view are outside the original viewing area. When the synthesized image is not acceptable, we can simply select another center to reconstruct a new IPO. Consequently, we will obtain multiple IPOs for a widely spread 3-D scene which can keep the 3-D data reasonably accurate and reduce the amount of memory required to save an infinitely large 3-D space to a manageable size. The idea of representing 3-D environments using multiple IPOs is similar to the QuickTime VR technique of Apple, Inc. As in the QuickTime VR technique, the IPOs can be used to provide panoramic views of a 3-D scene. But they also can be used to synthesize images when the virtual camera moves around the centers of the IPOs, which cannot be achieved by using the QuickTime VR technique.

\section{AUTOMATIC 3-D RECONSTRUCTION}

\subsection{Visually Inconsistent Regions}

The schematic diagram of our active 3-D reconstruction process is shown in Fig. 3. We assume that the positions and orientations of the stereo cameras at any configuration of the binocular head are available for exploring and reconstructing the 3-D environment. In practice, this requirement can be achieved by using a well-calibrated active binocular head equipped with accurate position and orientation sensors, such as InterSense IS-900 CT, Fastrak, or Flock of Birds. That is, the parameters of the stereo cameras on the binocular head are known at any time instant, based on the kinematic model of the binocular head and the readings of the position and orientation sensors. Hence, we can adopt the asymptotic Bayesian estimation method, which assumes the camera parameters are known for each camera position.

To reconstruct the 3-D environment more efficiently, we do not apply the asymptotic Bayesian estimation to an image region unless it is necessary, or more precisely, unless it is a visually inconsistent region (which is defined below). If a set of camera parameters

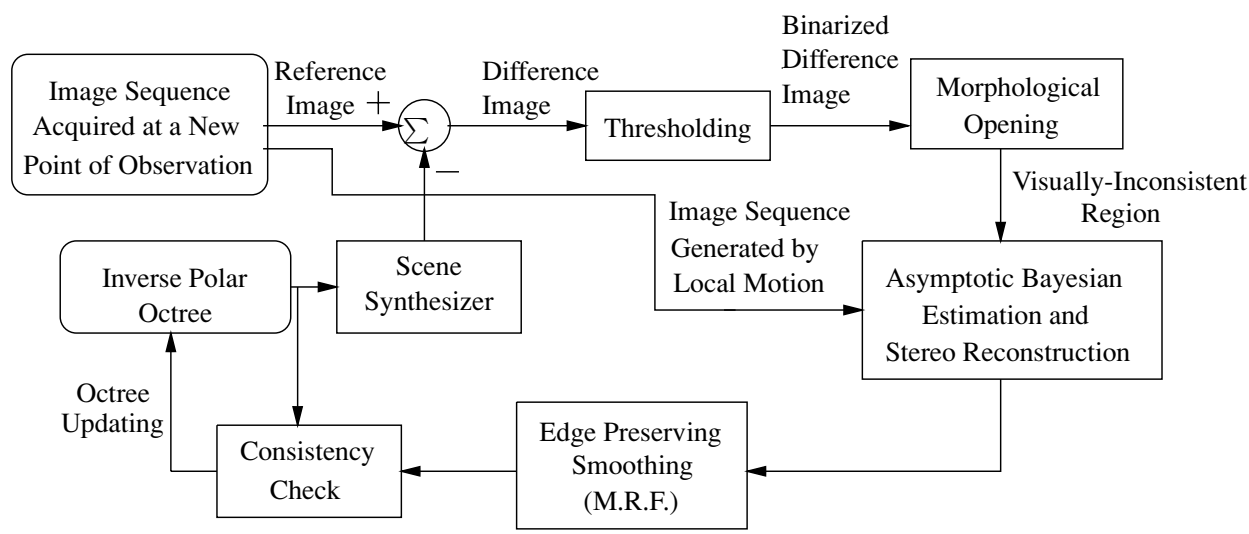

FIG. 3. Schematic diagram of the automatic 3-D reconstruction process. 
are specified, we can synthesize images according to the current world model stored in the IPO by using ray tracing techniques (refer to the Appendix). Next, the synthesized image is subtracted from the observed image to obtain a difference image. The difference image is thresholded into binary image format and then filtered by using the morphological opening to remove noise. The active pixels in the filtered binary image can be grouped into regions indicating where the depth information is either incorrect or not available. The detected regions will be referred to as the visually inconsistent regions (VIRs) because the observed image is visually inconsistent with the synthesized image according to the IPO. At the very beginning, the IPO contains no valid data. Hence, the whole image is a VIR and must be processed to estimate 3-D depth as described in the next section. Once some 3-D depth estimates are stored in the IPO, only the VIRs must be processed.

\subsection{Depth Estimation}

To estimate the 3-D depth, we first partition the VIRs into small blocks and then assume that each block in the left image is projected from a 3-D planar patch having a constant depth. The depth estimation method we used in this work is mainly the asymptotic Bayesian estimation method proposed by Hung et al. [26], which is adapted for active vision purpose and is described briefly in the following. At first, a reference image is acquired by using the left camera at an initial pose. The reference image will also be used in integrating new 3-D observations into the IPO (refer to Section 3.4); therefore, it is kept in the memory during the whole 3-D reconstruction process. Suppose that the depth, $d$, of block $P$ in the VIRs of the reference image is to be estimated. Let the initial estimate of the reciprocal variance of $d$ be $\Phi_{1}=0$, since we do not have any information about $d$ yet. To obtain the correct stereo correspondence of $P$ in the right image, we first move the left camera locally and incrementally to compute a rough estimate of the depth of $P$ (the way we determine the path of the local motion will be described in the next section). Now, suppose that we have obtained a sequence of images all acquired by using the left camera, denoted by $I_{1}, I_{2}, \ldots$, where $I_{1}$ is the reference image of this image sequence and the other images are acquired during the local camera motion. Since the binocular head is well calibrated, we have the relative geometric relation (i.e., the relative camera position and orientation) of the image pair $\left(I_{1}, I_{n}\right)$, where $n \geq 2$. Based on the geometric relation and a given depth estimate $\hat{d}$, we can compute, for each pixel in a block $P \in I_{1}$, the corresponding image point in the $n$th image. For convenience, let $s$ be a 2-D image point in image block $P \in I_{1}$ and let $u_{n}(s, \hat{d})$ denote its corresponding image point in the new image, i.e., (the $n$th image), as shown in Fig. 4 . The depth of the image patch, $d$, can be refined by minimizing the following objective function,

$$
J_{n}(d)=\frac{1}{2}\left(d-\hat{d}_{n-1}\right)^{t} \Phi_{n-1}\left(d-\hat{d}_{n-1}\right)+\frac{1}{2} \sum_{s \in P}\left[I_{n}\left(u_{n}(s, d)\right)-I_{1}(s)\right]^{2},
$$

where $I_{1}(s)$ and $I_{n}\left(u_{n}(s, d)\right)$ are the intensity value of pixel $s$ in image 1 and the intensity value of pixel $u_{n}(s, d)$ in image $n$, respectively, and $\Phi_{n-1}$ denotes the reciprocal variance of the estimated depth $\hat{d}_{n-1}$ given images $1,2, \ldots, n-1$. The reciprocal variance can be updated by using the following equation:

$$
\Phi_{n}=\Phi_{n-1}+\left.\frac{\partial^{2}}{\partial d^{2}}\left\{\frac{1}{2} \sum_{s \in P}\left[I_{n}\left(u_{n}(s, d)\right)-I_{1}(s)\right]^{2}\right\}\right|_{d=\hat{d}_{n}}
$$




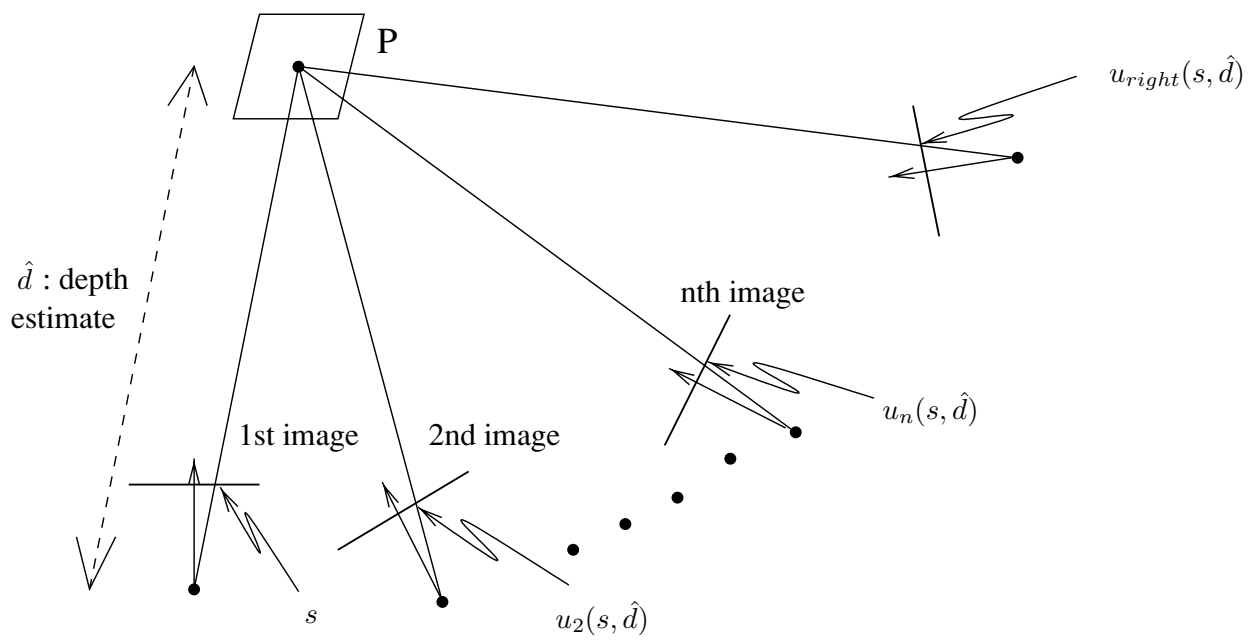

FIG. 4. The stereo correspondence of pixel $s$ computed by using the estimated depth $\hat{d}$ and the relative camera geometric parameters.

The asymptotic Bayesian process for estimating the depth of an image patch $P$ is summarized in the following.

1. Set $\Phi_{1}=0, n=1$, and $d_{1}=\infty$.

2. Acquire the reference image $I_{1}$ using the left camera.

3. Set $n=n+1$.

4. Acquire the $n$th image using the left camera.

5. Compute $\hat{d}_{n}$ by minimizing the error function in (2) using a gradient descent method.

6. Update $\Phi_{n}$ with (3).

7. Discard image $I_{n}$.

8. Repeat steps 3-7 until the estimation error is satisfactorily small.

More details about the asymptotic Bayesian method can be found in [26].

The local motion and asymptotic Bayesian estimation method can be repeated until we obtain accurate enough depth estimates; however, recall that the goal of performing local motion is to get a rough estimate of the depth value for determining stereo correspondences more accurately. Since the depth estimation error using local motion is proportional to the length of the effective movement, the local motion can be terminated when the length of the length of movement is greater than some value. According to our analysis (refer to [29], $50 \mathrm{~mm}$ of incremental local motion can reduce the depth uncertainty to such a level that the search region for stereo correspondence is less than 10 pixels in our setup; i.e.,

$$
\left|u_{n}\left(s, \hat{d}_{n}\right)-u_{n}\left(s, d_{\text {true }}\right)\right| \leq 5,
$$

where $d_{\text {true }}$ is the true value of the depth of block $P$. Therefore, once the effective movement length of the incremental local motion is greater than $50 \mathrm{~mm}$, our system will use the image taken by the right camera as the new input image of the asymptotic Bayesian estimation process (i.e., a big jump) and perform an exhaustive search for the minima of (2) along the epipolar line in the 10-pixel search region centered at $u_{\text {right }}\left(s, \hat{d}_{n}\right)$. Then, a gradient descent search is performed to further refine the depth estimate. After the depth estimates 


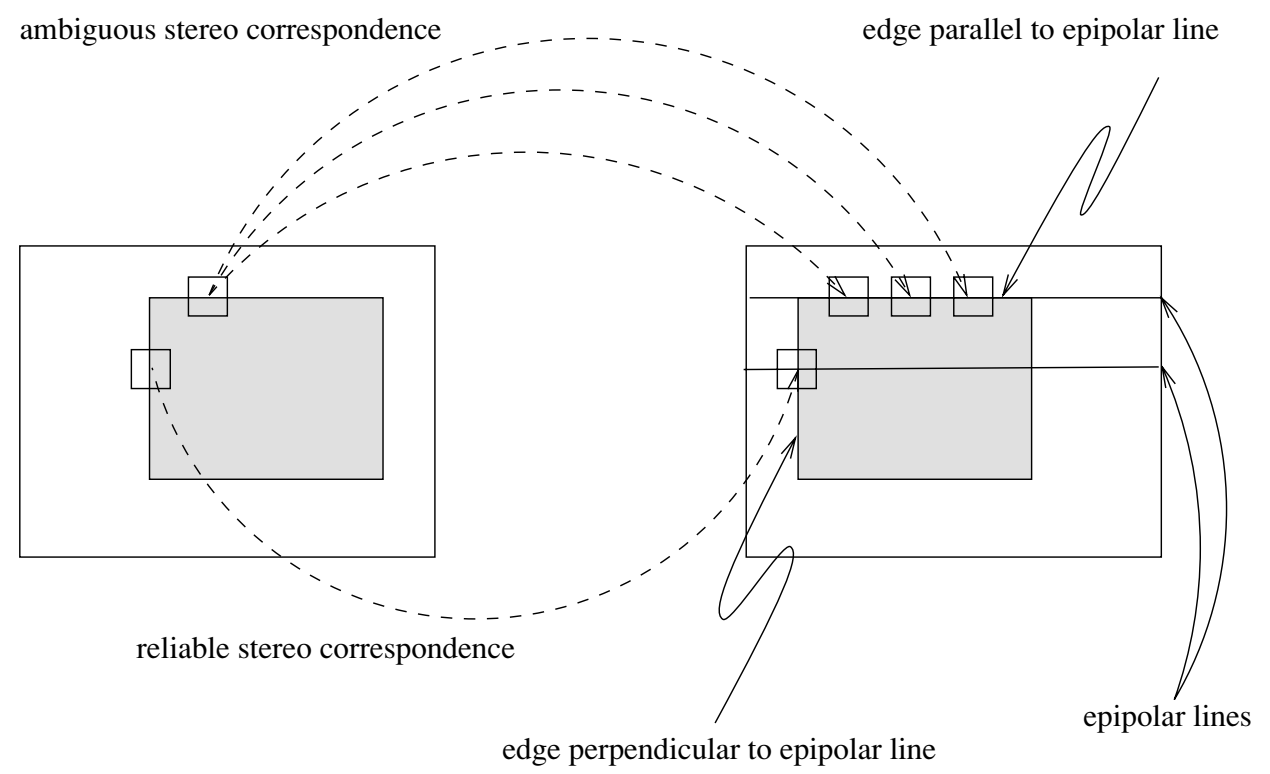

FIG. 5. Edge parallel to the epipolar line will cause ambiguity in stereo matching.

of all the patches in the visually inconsistent regions are computed with the above process, Markov random fields can be used to smooth the depth map while preserving the depth discontinuity [30]. The smoothed 3-D data are integrated into the IPO using the method described in Section 3.4. The active binocular head then moves to another new station and the depth estimation procedure repeats for the VIRs until the size of all VIRs are small enough.

\section{Occupied Voxels}

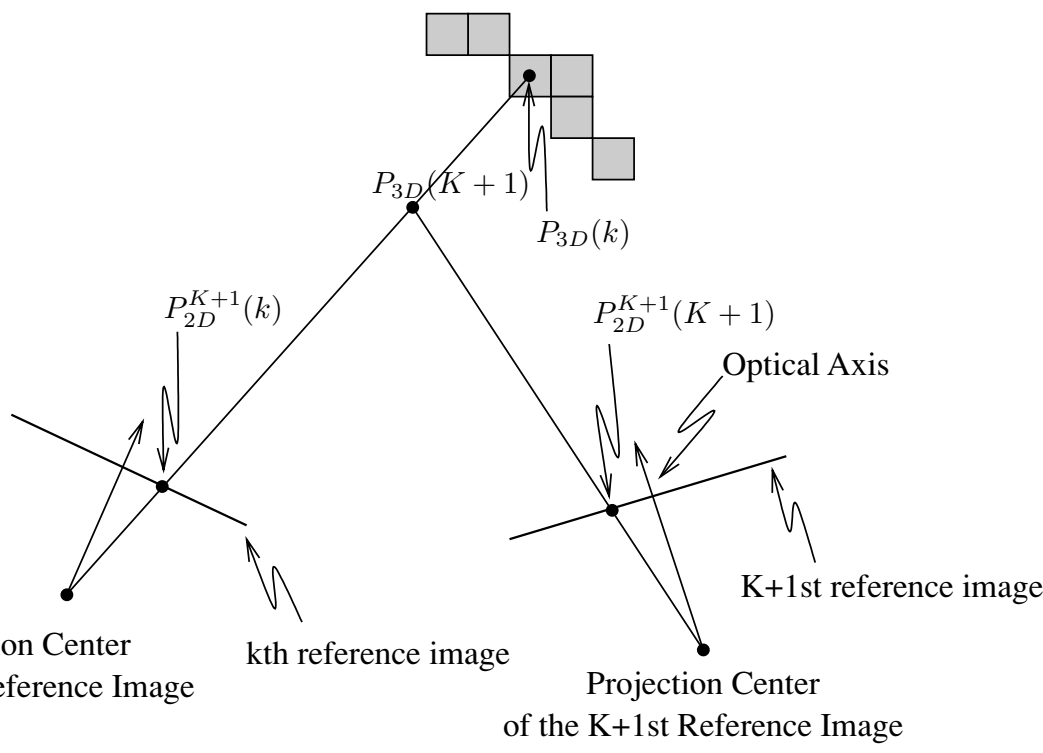

FIG. 6. Geometrically incompatible 3-D data. Notice that $P_{3 D}(k)$ is occluded by $P_{3 D}(K+1)$ when observed from the projection center of the $k$ th reference image. 


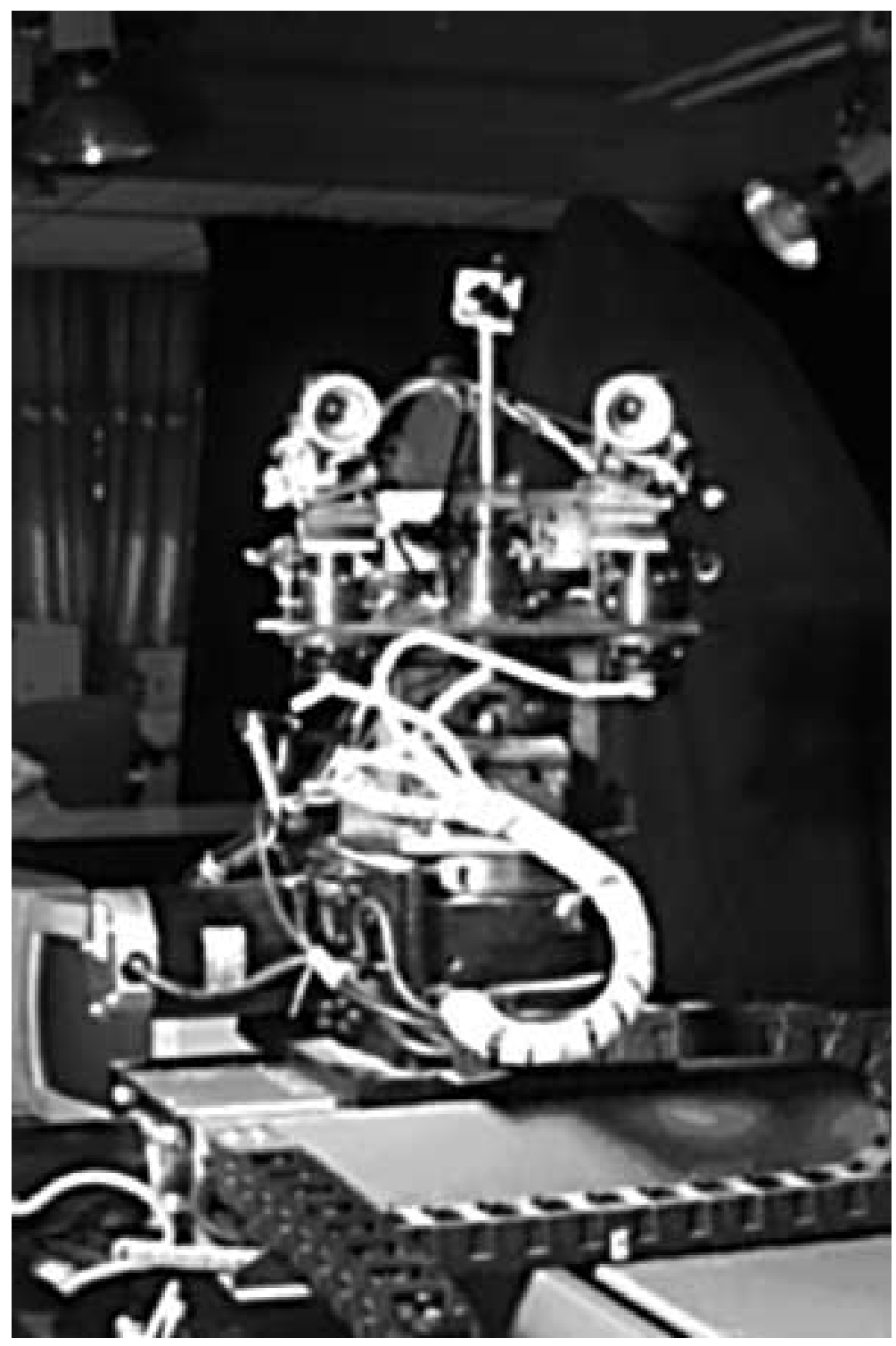

FIG. 7. The active binocular head (the IIS head) used in the experiments.

\subsection{Path Planning for Local Motion}

Having a well-calibrated active binocular head, we are able to control the cameras to move along a path which can reduce the ambiguity level of stereo matching. Our path planning method is based on the following observation: when performing stereo matching, we can determine the stereo correspondence more easily and reliably if the edge orientation is perpendicular to the epipolar line, as shown in Fig. 5. On the other hand, if the edge orientation is parallel to the epipolar line, then finding stereo correspondence is an ill-posed problem. To eliminate the ambiguity in stereo matching, the local motion is selected to form epipolar lines which are perpendicular to most edges having highly uncertain depth estimates. The following procedure describes the way we determine the local motion: 

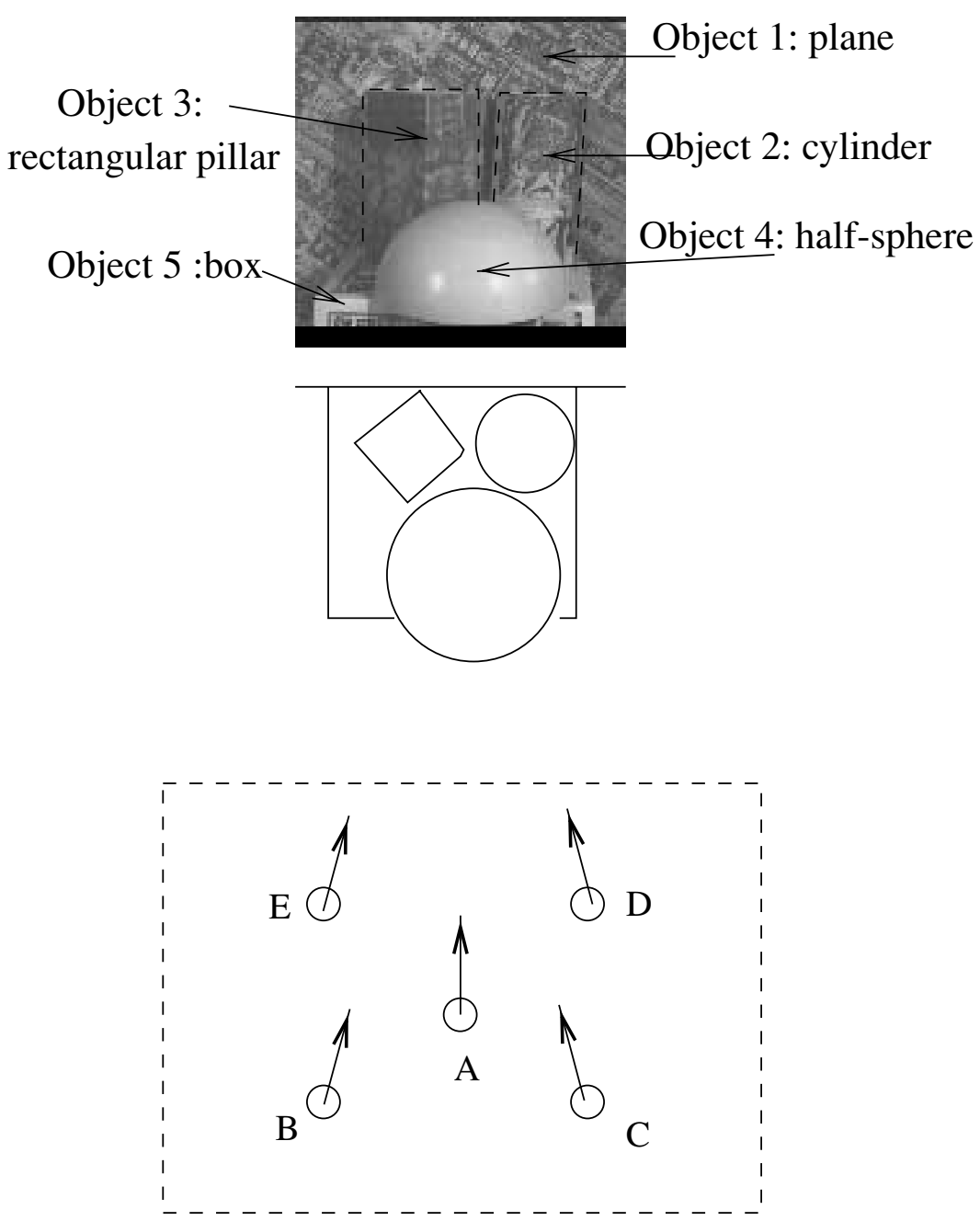

FIG. 8. The distribution of the five points of view used to acquire the images as shown in Fig. 9.

1. Perform Sobel edge detection on the new input image and record the orientations $\theta_{j}$ of each edge pixel $j$.

2 . For each edge pixel $j$, get its reciprocal variance value, $\Phi_{j}$, determined in the asymptotic Bayesian process. Notice that a larger value of $\Phi_{j}$ indicates that the depth estimate of pixel $j$ is more reliable because $\frac{1}{\Phi_{j}}$ is the variance of the depth estimate of pixel $j$.

3. Compute the average edge orientation weighted by its variance value as follows:

$$
\Theta=\frac{\sum_{j: \Phi_{j}>0}\left(\frac{\theta_{j}}{\Phi_{j}}\right)}{\sum_{j: \Phi_{j}>0}\left(\frac{1}{\Phi_{j}}\right)} .
$$

Notice that in (5), edge orientations corresponding to depth estimates of higher uncertainty will be weighted more heavily. 


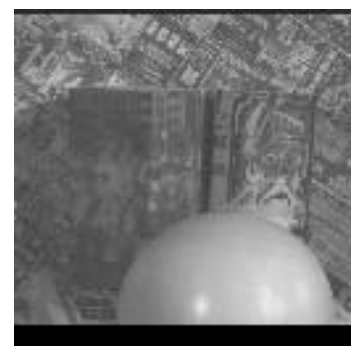

E

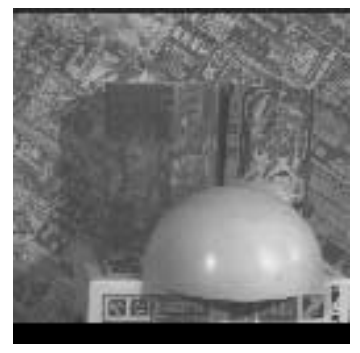

B

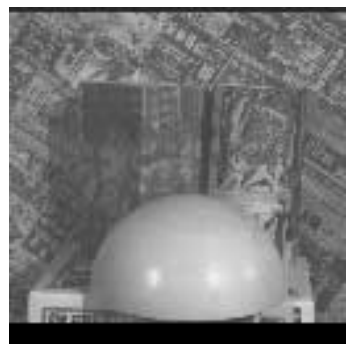

A

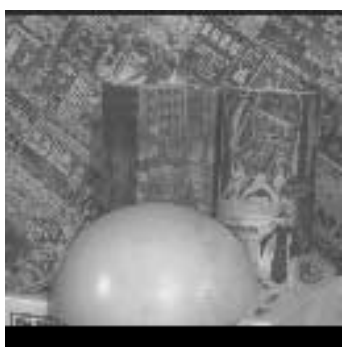

D

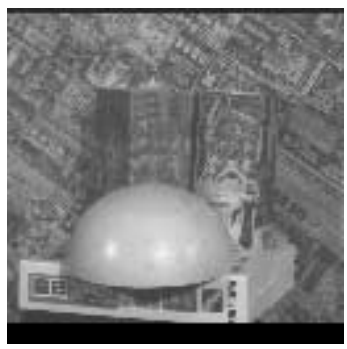

C

FIG. 9. Five reference images acquired by the left camera at the five points of view as shown in Fig. 8.

4. Compute the horizontal and vertical motion components, $H_{\text {move }}$ and $V_{\text {move }}$, of the camera

$$
H_{\text {move }}=\Delta_{H} \cos \left(\Theta+\frac{\pi}{2}\right)
$$

and

$$
V_{\text {move }}=\Delta_{V} \sin \left(\Theta+\frac{\pi}{2}\right),
$$

where $\Delta_{H}$ and $\Delta_{V}$ are two predetermined constants specifying the step size of each movement.

\subsection{Consistency Check for a New 3-D Observation}

Suppose that we have moved the active binocular head to $K+1$ different stations, that we have collected $K+1$ reference images and the corresponding camera parameters at the $K+1$ stations, that the 3-D data observed at the first $K$ stations have been integrated into the IPO, and that the 3-D data observed at the $(K+1)$ st station is to be integrated into the IPO. Only those new 3-D data which are consistent with the old data were integrated into the IPO. Rules for checking the consistency are described below.

Suppose a new depth estimate, whose 3 -D coordinates are $p_{3 D}(K+1)$, is considered to be integrated into the IPO. Let $p_{2 D}^{K+1}(K+1)$ be the image location of $p_{3 D}(K+1)$ on the $(K+1)$ st reference image, and let $p_{2 D}^{K+1}(k)$ be the projected 2-D image location of 


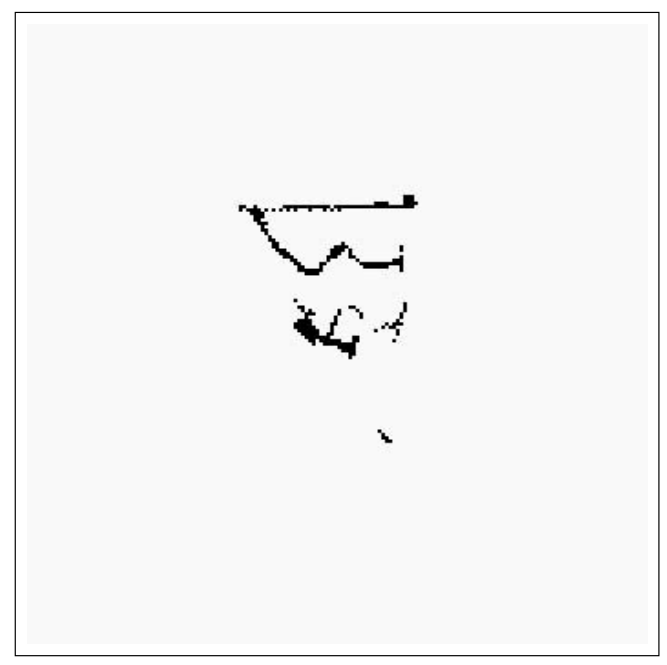

(a)

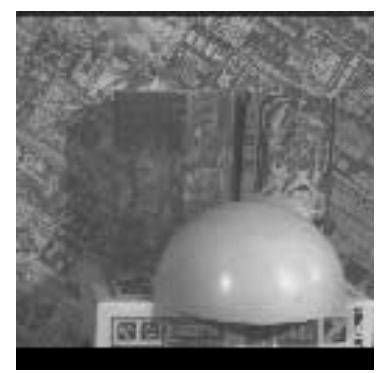

(c)

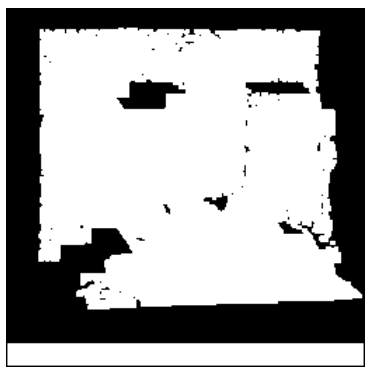

(d)

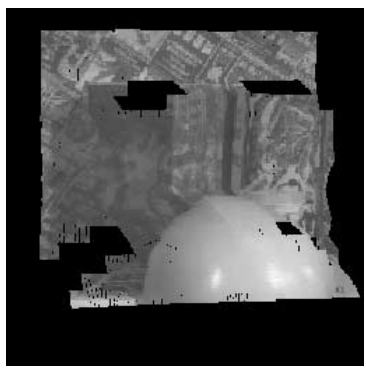

(b)

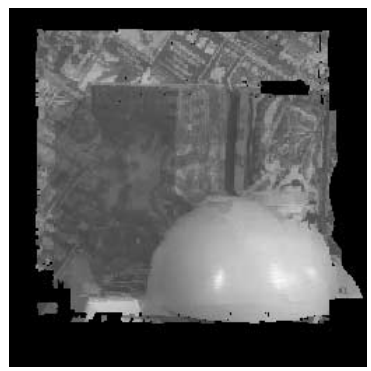

(e)

FIG. 10. (a) The 3-D data contained in the octree reconstructed at viewpoint $A$ were projected to a plane parallel to the ground. (b) An image synthesized at viewpoint $B$ using the octree data shown in (a). (c) The observed image at viewpoint $B$. (d) The difference image shows the virtually inconsistent regions. (e) An image synthesized at viewpoint $B$ using the octree data updated by the depth estimates in the visually inconsistent regions of (d).

$p_{3 D}(K+1)$ on the $k$ th reference image. By back-projecting $p_{2 D}^{K+1}(k)$ into a 3-D ray and computing the first intersection point of the 3-D ray and the occupied voxel in the IPO, we have $p_{3 D}(k)$. We say that the new observation $p_{3 D}(K+1)$ is geometrically compatible with the $k$ th observation if $p_{3 D}(k)$ is not occluded by $p_{3 D}(K+1)$ when observed from the projection center of the $k$ th reference image (see Fig. 6 for an example of geometrically incompatible 3-D data). If $p_{3 D}(K+1)$ is geometrically incompatible with the $k$ th reference image, then we further check if its color (or gray level) is compatible with that of the $k$ th observation; i.e.,

$$
\left|I_{K+1}\left(p_{2 D}^{K+1}(K+1)\right)-I_{k}\left(p_{2 D}^{K+1}(k)\right)\right|<\tau_{C},
$$

where $\tau_{C}$ is a given threshold value. If $p_{3 D}(K+1)$ is either geometrically compatible or color-compatible with the $k$ th reference image, then we say that $p_{3 D}(K+1)$ is compatible with the $k$ th reference image, since, in either case, adding the 3 -D point, $p_{3 D}(K+1)$, into the IPO will not cause visual inconsistency between reference image $K+1$ and $k$. 


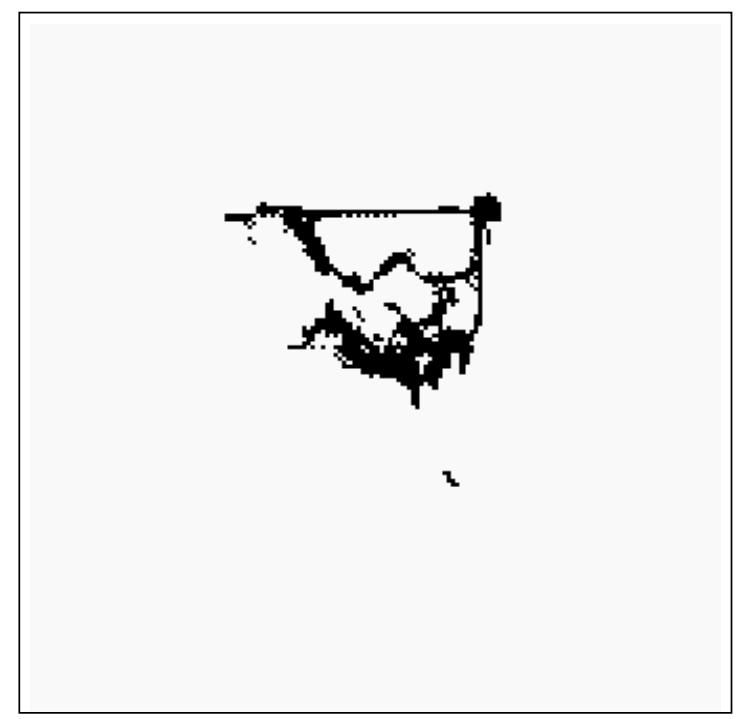

(a)

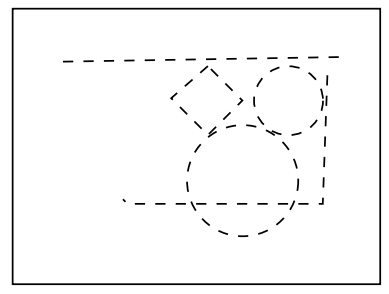

(b)

FIG. 11. (a) The 3-D data contained in the octree reconstructed at the five viewpoints were projected to a plane parallel to the ground. (c) The corresponding objects of (a).

If more than two-thirds of the reference images are compatible with the new observation, then $p_{3 D}(K+1)$ is said to be largely consistent with the previous observations and is used to update the IPO. If the new observation is not largely consistent with the previous observations, it is discarded. After a new observation $p_{3 D}(K+1)$ is determined to be largely consistent with the previous observations, we remove those old 3-D voxel data which occlude $p_{3 D}(K+1)$ on the projection center of the $(K+1)$ st reference image.

Notice that, in the above-mentioned consistency check process, we did not use the uncertainty information, i.e., $\Phi_{j}^{-1}$, a by-product of the asymptotic Bayesian estimation method (refer to Section 3.2), of the 3-D estimates. This is because the uncertainty measurement is valid only when the 3-D estimation error is small. When the 3-D estimation error is small, it is unlikely to fail in the consistency check process. Therefore, we did not use the uncertainty information in the consistency check. As to the data integration, even if the estimated 3-D data are largely consistent with the previous observations, we still cannot ensure that the 3-D data are likely to be very accurate, and hence, we also ignore the uncertainty information of the 3-D estimates in the data integration process. 


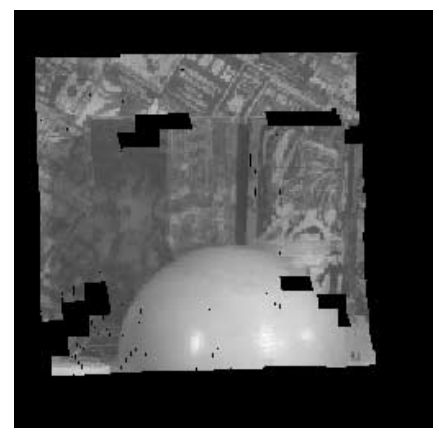

(a)

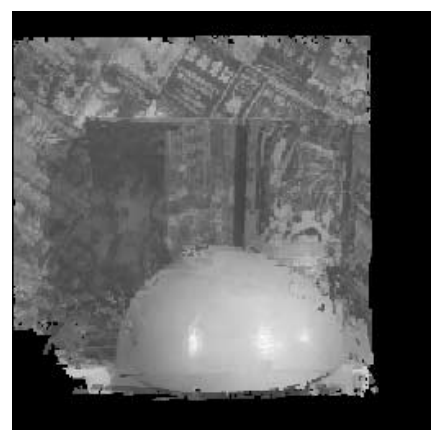

(c)

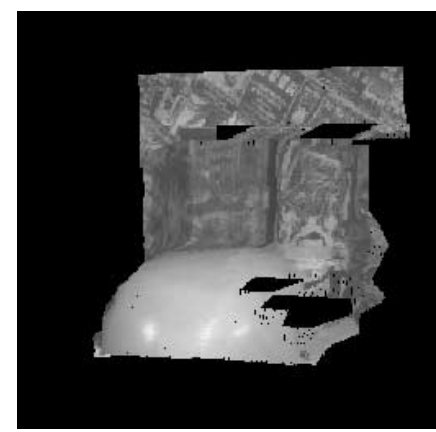

(b)

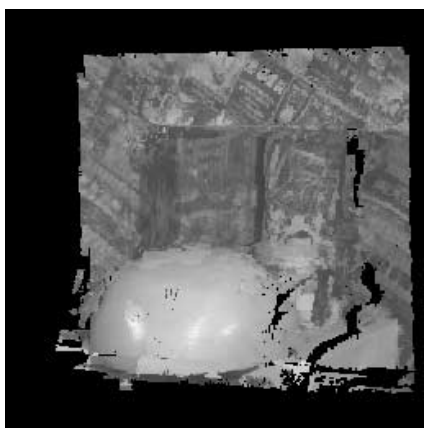

(d)

FIG. 12. (a, b) The images synthesized at a virtual viewpoint $F$, which is located between viewpoints $A, B$, and virtual viewpoint $G$, which is located at the right side of viewpoint $C$ by using the octree data reconstructed after observations were taken at viewpoint $A$. (c, d) The images synthesized at virtual viewpoints $F$ and $G$ by using the octree data reconstructed after observations were taken at viewpoints $A-E$.

\section{EXPERIMENTS}

In the experiments, a well-calibrated binocular head [12] as shown in Fig. 7 was used to acquire stereo image sequences. The binocular head is mounted on an $\mathrm{X}-\mathrm{Y}$ table which is used to emulate a mobile robot platform. Two experiments were conducted to test the proposed active vision algorithm. In the first experiment, the target scene consisted of five objects. Four of them have textured surfaces, namely, a planar background, a cylinder, a rectangular pillar, and a box, whereas the other object is a textureless hemisphere. The relative positions of the five objects and five viewpoints for the 3-D measurement chosen in advance are illustrated in Fig. 8. The reference images acquired at those five viewpoints by the left camera are shown in Fig. 9. At each viewpoint, a sequence of local movements is performed automatically to estimate the 3-D information corresponding to the VIRs by using the asymptotic Bayesian method. At the very beginning, the inverse polar octree contained no valid data. Hence, the whole image was visually inconsistent and had to be processed to estimate 3-D depth. Figure 10a shows a bird's eye view of the world model reconstructed at viewpoint $A$. In Fig. 10a, we can find the contours of the rectangular pillar and part of the cylinder. When the active binocular head was driven to viewpoint $B$, an image was synthesized at viewpoint $B$ according to the world model reconstructed at viewpoint $A$. The synthesized image is shown in Fig. 10b, whereas the observed image at viewpoint $B$ is 


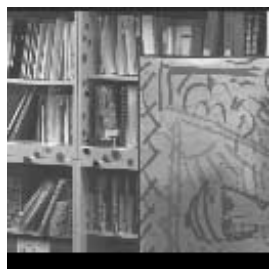

A1

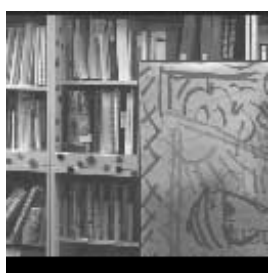

B1

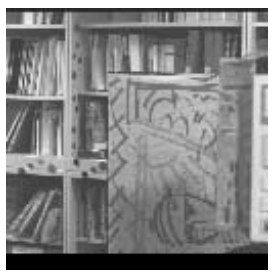

C1

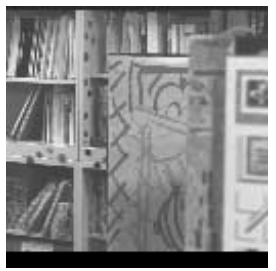

D1

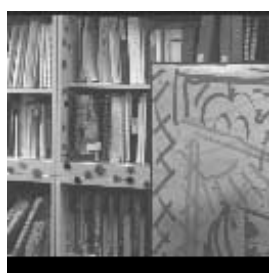

E1

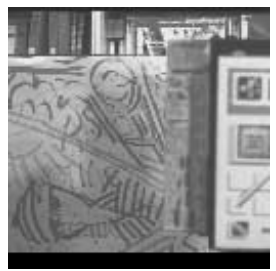

A2

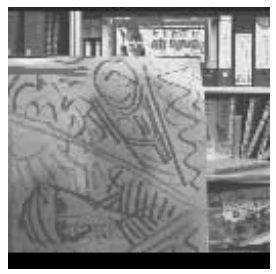

B2

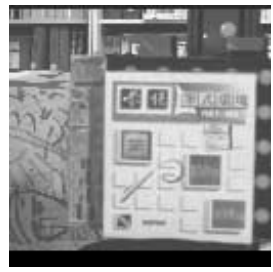

C2

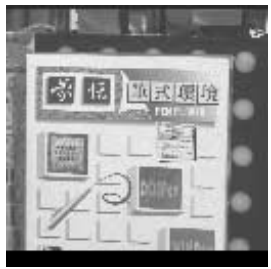

D2

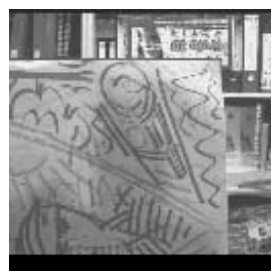

E2

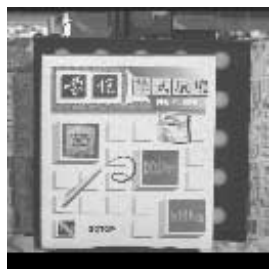

A3

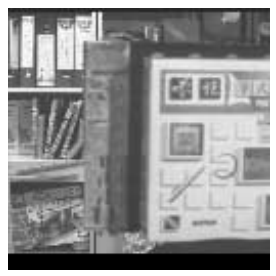

B3

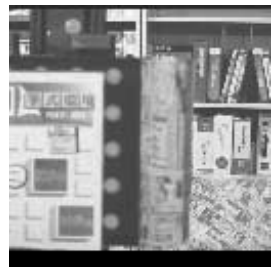

C3

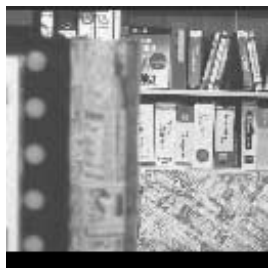

D3

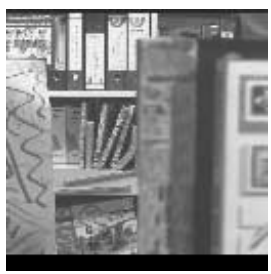

E3

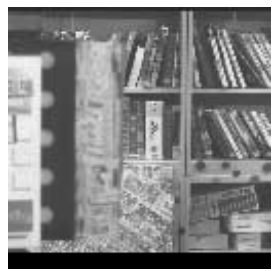

A4

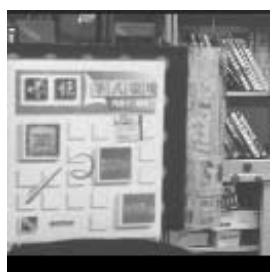

B4

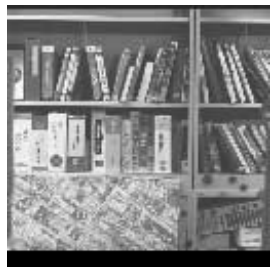

C4

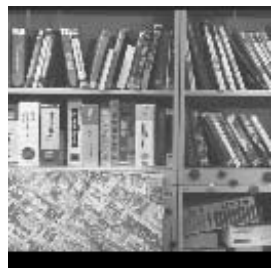

D4

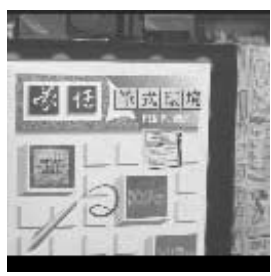

E4

FIG. 13. Twenty reference images acquired by the left camera at the 20 viewpoints, $A 1, A 2$, etc., and $E 4$, illustrated in Fig. 14.

shown in Fig. 10c. The VIRs (marked with black pixels) computed based on Figs. 10b and 10c are shown in Fig. 10d. Only the 3-D data of the VIRs needed to be estimated/reestimated using the asymptotic Bayesian method. The estimation results were then used to update the world model. Figure 10e shows the synthesized image at viewpoint $B$ rendered by using the updated world model. Figure 11 shows a bird's eye view of the reconstructed world 


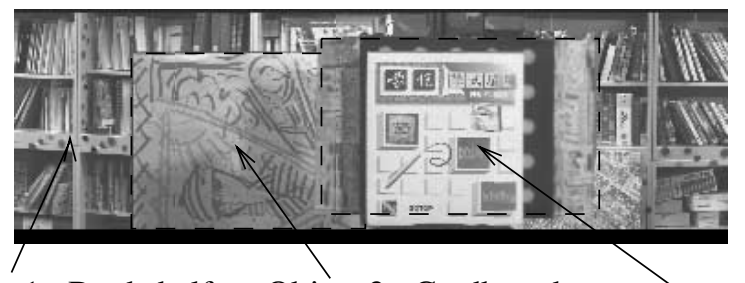

Object 1 : Bookshelf Object $2:$ Cardboard Object $3:$ Box

Object 1

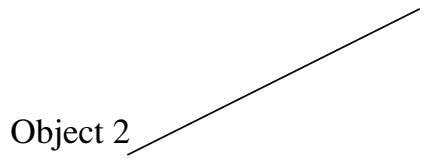

Object 3

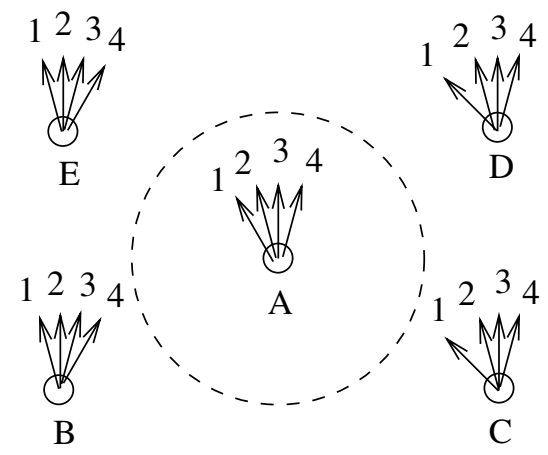

FIG. 14. The relative positions of the objects seen in the scene and the 20 viewpoints, $A 1, A 2$, etc., and $E 4$, used for taking observations.

model after all the images observed at the five viewpoints $A-E$ have been processed. Figure 12 shows some intermediate and final results in the reconstruction process. Those images were synthesized at two virtual viewpoints $F$ and $G$, where virtual viewpoint $F$ is located between viewpoints $A$ and $B$, and virtual viewpoint $G$ is located at the right side 


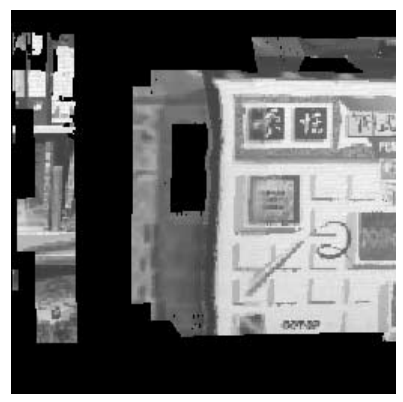

(a)

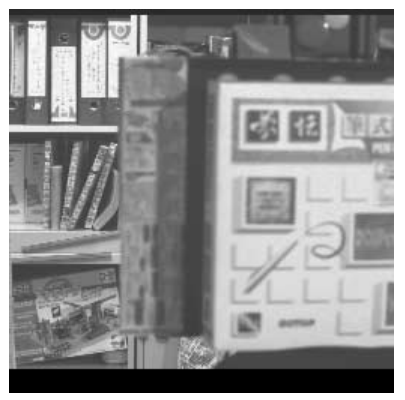

(b)

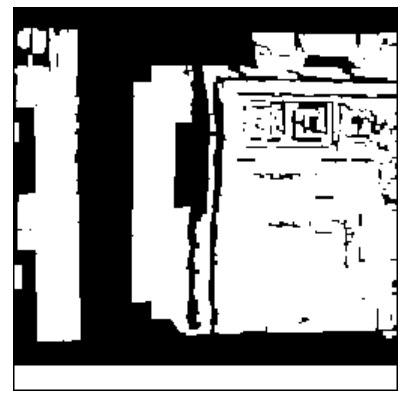

(c)

FIG. 15. (a) Synthesized image. (b) Observed image. (c) Visually inconsistent regions (those regions marked in black).

of viewpoint $C$. Figures $12 \mathrm{a}$ and $12 \mathrm{~b}$ show the images synthesized at virtual viewpoints $F$ and $G$ according to the world model reconstructed using only the observations made at viewpoint $A$, respectively. Figures $12 \mathrm{c}$ and $12 \mathrm{~d}$ show the images synthesized according to the world model reconstructed after all the images acquired at the five viewpoints are processed. Notice that the reconstructed 3-D model of the scene became more complete as more and more images were processed.

In the second experiment, we show how a complex scene in our laboratory can be reconstructed with the active binocular head. First, 20 viewpoints for 3-D reconstruction were chosen in advance. The reference images acquired by the left camera at the 20 viewpoints are shown in Fig. 13. The relative position of the objects and the 20 viewpoints are shown
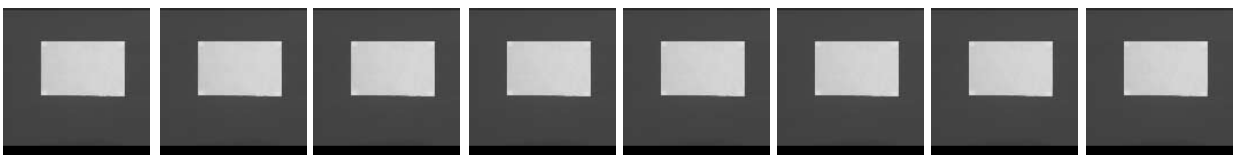

(a)
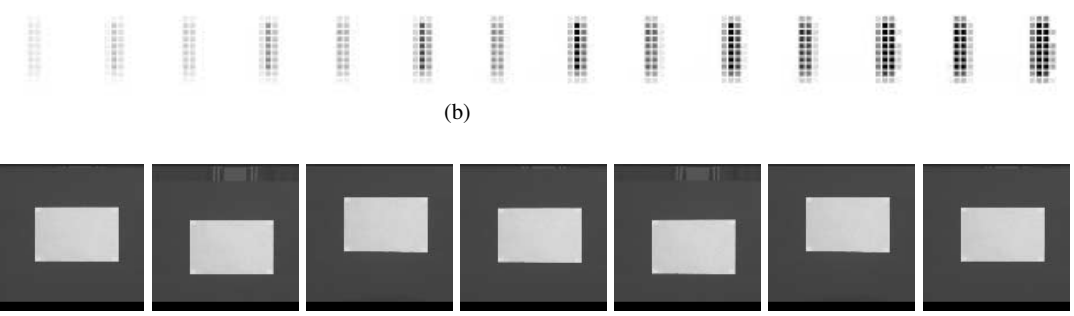

(b)
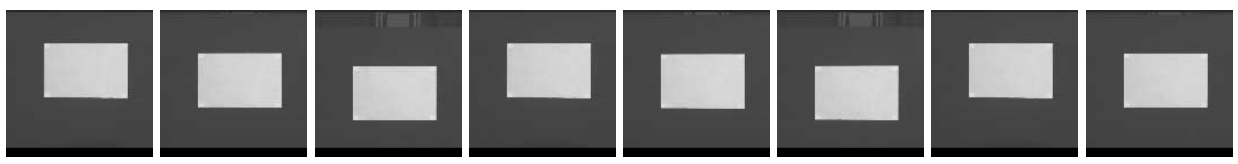

(c)

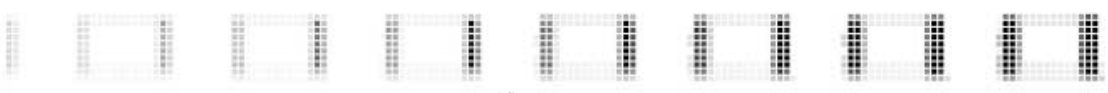

(d)

FIG. 16. (a) A sequence of images acquired when the active camera was moved horizontally. (b) The reciprocal variance value $\Phi$ for each image in (a). (c) Images acquired in a sequence of local camera motions whose path is determined online. (d) The reciprocal variance values $\Phi$ of the horizontal edges increased when the local motion planner functions. 


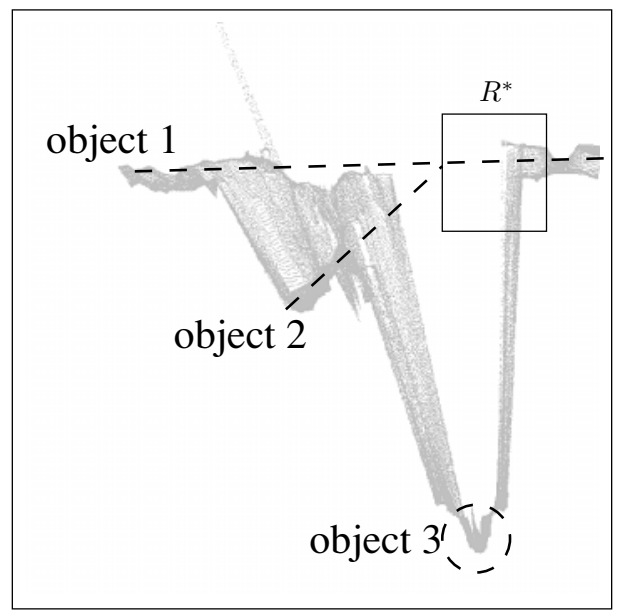

(a)

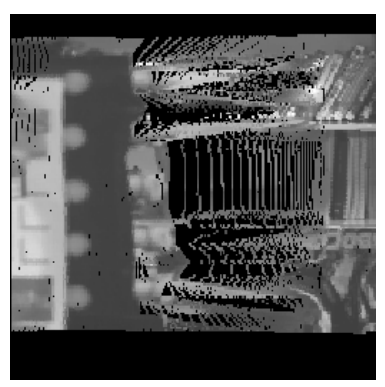

(c)

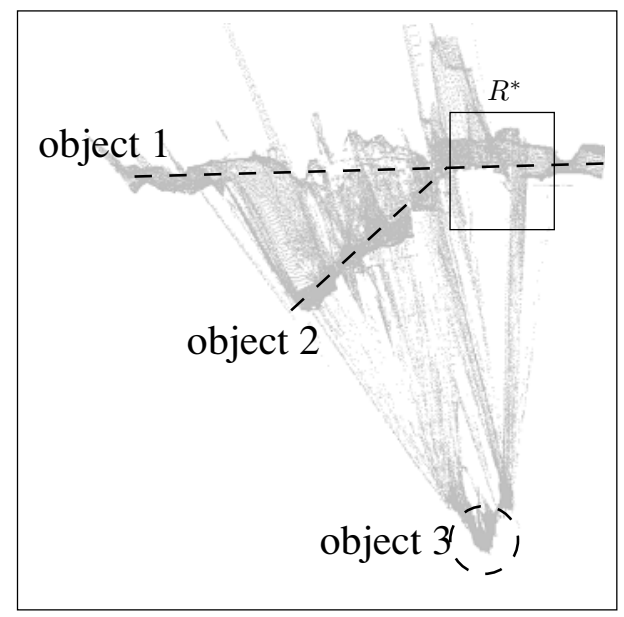

(b)

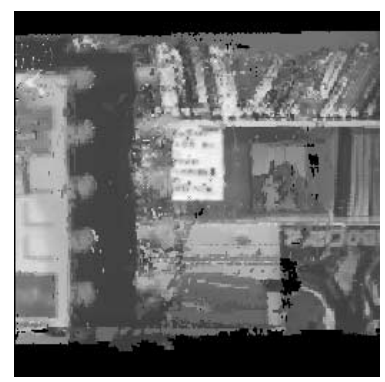

(d)

FIG. 17. (a) The $3-\mathrm{D}$ data contained in the octree reconstructed at viewpoints $A 1-A 4$ were projected to a plane parallel to the ground. (b) The 3-D data contained in the octree reconstructed at the 12 viewpoints were projected to a plane parallel to the ground. (c) An image synthesized at a viewpoint, $V$, located between viewpoints $A$ and $C$ by using the octree data shown in (a). (d) An image synthesized at the viewpoint, $V$, by using the octree data shown in (b).

in Fig. 14, where Object 1 is a bookshelf in the background, Object 2 is a textured cardboard, and Object 3 is a box. At each of the 20 viewpoints, a sequence of local movements is performed to estimate the depth value of the VIRs by using the asymptotic Bayesian method. Figure 15 shows typical images of synthesized and observed images, as well as the corresponding computed VIRs. Figure 16 shows an image sequence (for illustrating the effect of our local motion planner) acquired along two paths of local motion, a purely horizontal path (shown in Figs. 16a and 16b) and a sequence of motion determined by the method described in Section 3.3 (shown in Figs. 16c and 16d). Notice that, in Fig. 16d, the reciprocal variance value increased from left to right as more and more images were acquired and processed. Also, the computed local motion drove the camera to move both vertically and horizontally to reduce the ambiguity of stereo matching. Since the vertical camera motion could not be generated by the $\mathrm{X}-\mathrm{Y}$ translation table, we moved the tilt joint to generate an equivalent vertical camera motion, which was possible (although quite limited) because the lens center of the camera was located a distance off the rotation axis of the tilt joint. 


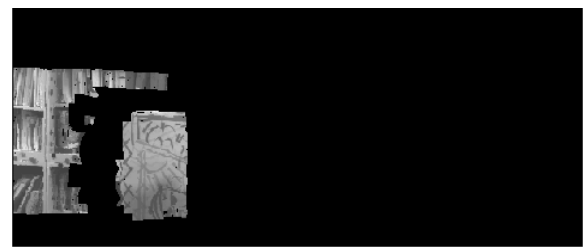

(a)

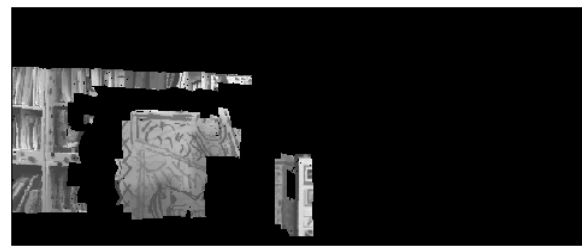

(b)

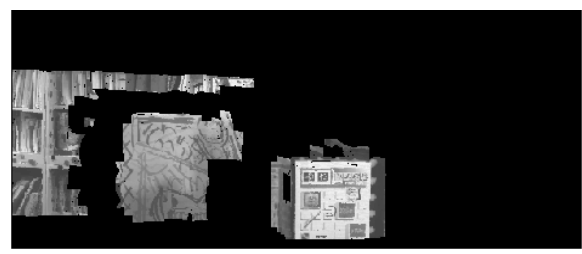

(c)

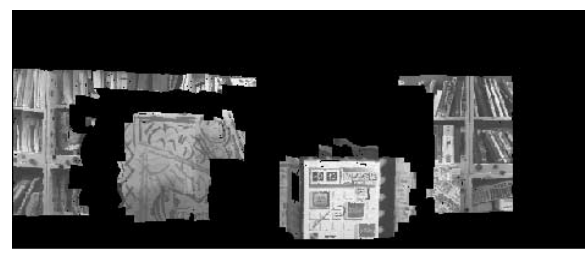

(d)

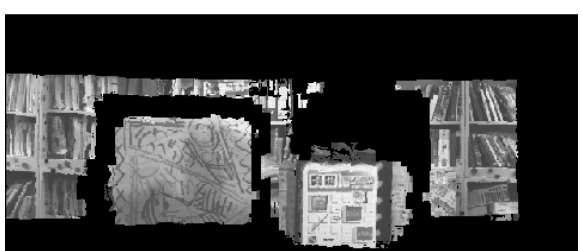

(e)

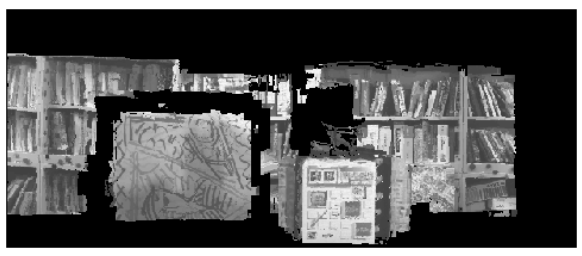

(f)

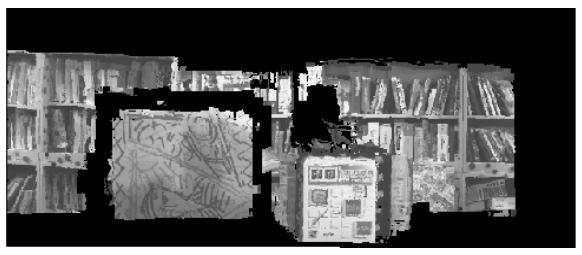

(g)

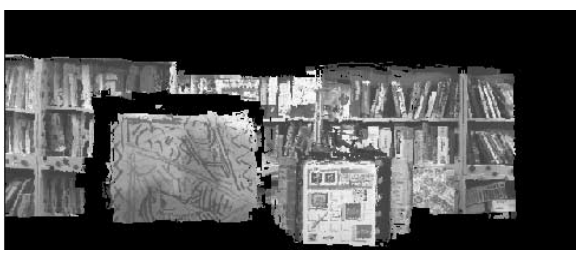

(h)

FIG. 18. Synthesized images from a virtual viewpoint slightly overlooking the scene, where (a)-(h) are synthesized by using the IPO reconstructed after observations were taken at viewpoints $A 1, A 1-A 2, A 1-A 3$, $A 1-A 4, A 1-B 4, A 1-C 4, A 1-D 4$, and $A 1-E 4$, respectively.

The 3-D data obtained by using the images taken at viewpoints $A 1-A 4$ are shown in Fig. 17a. Notice that when observed from viewpoints $A 1-A 4$, part of the bookshelf, i.e., region $R^{*}$ marked in this figure, is occluded by Object 3. Figure $17 \mathrm{c}$ shows that an image synthesized at a virtual viewpoint, which is located between viewpoints $A$ and $C$, will contain several "holes" (black image regions) because the 3-D information of region $R^{*}$ is still not valid. However, after all the images observed at the 20 viewpoints are used to update the IPO, the 3-D structure becomes more complete, as shown in Fig. 17b, and the synthesized image based on the updated 3-D structure looks much better (most of the "holes" have been patched), as shown in Fig. 17d. Notice that during the asymptotic Bayesian process, constant depth values were assumed for each square image block (refer to Section 3.2). Next, the estimated depth values were interpolated and smoothed to obtain smooth 3-D surface, and the side effect of the interpolation process was that many undesired voxels between Objects 1, 2, and 3 are generated. However, these undesired voxels can be 


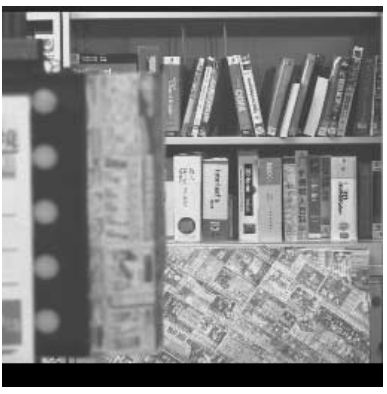

(a)

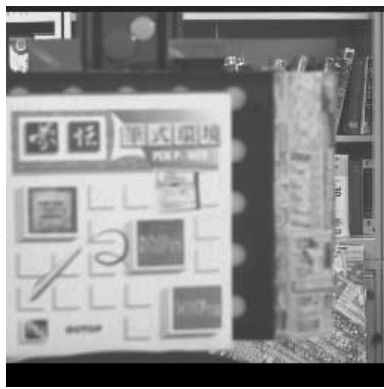

(c)

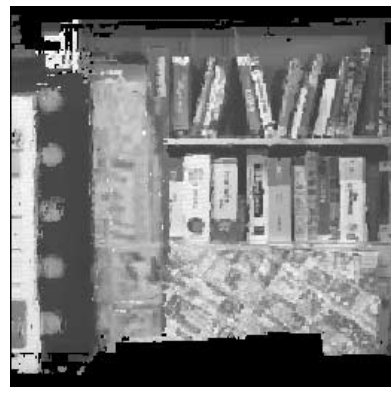

(b)

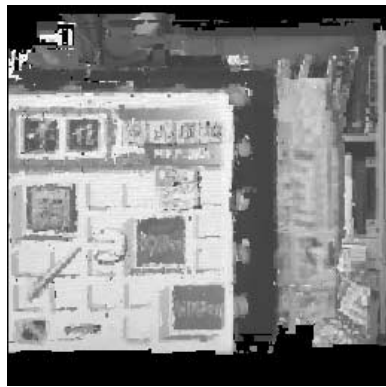

$(d)$

FIG. 19. (a) The real image captured at a viewpoint, $V$, which is located between viewpoints $A 4$ and $C 2$. (b) The synthetic image generated for $V$ by using the reconstructed IPO. (c) The real image captured by another viewpoint, $U$, located between viewpoints $A 3$ and $B 4$. (d) The synthetic image generated for $U$ by using the reconstructed IPO.

eliminated in the consistency check. As shown in Fig. 17b, many undesired voxels originally found in Fig. 17a have been removed. Figure 18 shows images synthesized at a viewpoint which is located above the real viewpoints and overlooking the scene. This figure shows that the reconstructed 3-D information becomes more complete and accurate as more images observed at different viewpoints were processed.

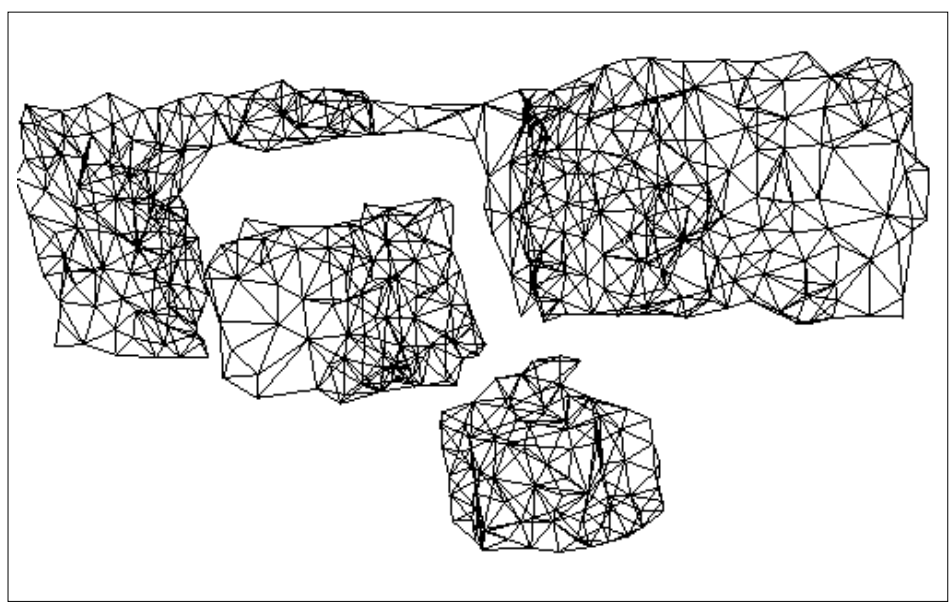

FIG. 20. The triangular meshes obtained by converting the $3-D$ data contained in the reconstructed IPO. 


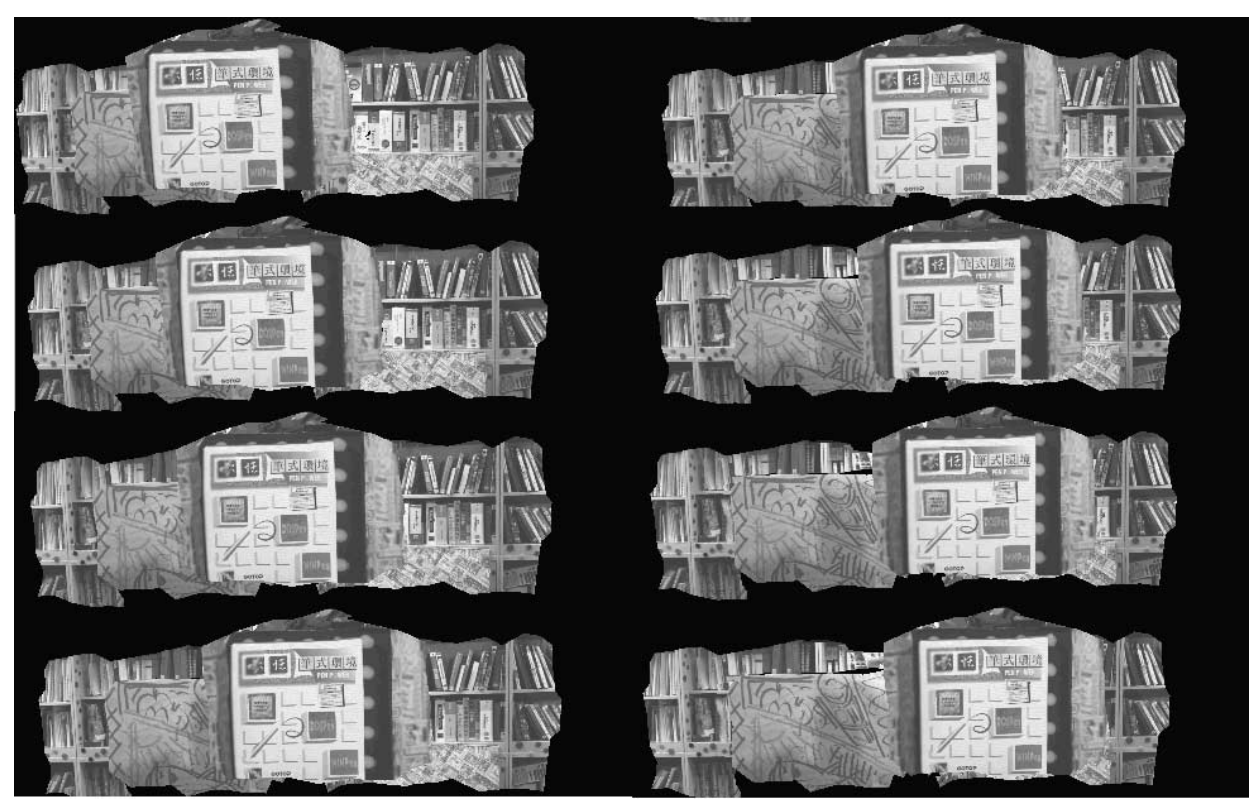

FIG. 21. An image sequence generated by using the texture-mapped triangular meshes.

For testing the reconstruction results, we manually selected two test viewpoints - one was located between viewpoints $A 4$ and $C 2$ and the other was located between viewpoints $A 3$ and $B 4$ - for acquiring test images. Figures $19 \mathrm{a}$ and $19 \mathrm{c}$ show the acquired test images and Figs. 19b and 19d show the synthesized images by using the reconstructed 3-D environment stored in the IPO. Notice that Figs. 19a and 19b and Figs. 19c and 19d look very similar, which means that the reconstruction results are visually consistent with the real image and thus can be used in some VR applications. The reconstructed data can also be converted to 3-D meshes for there are hardware graphic accelerators which can render texture-mapped 3-D meshes at video rate. Figure 20 shows the 3-D meshes converted from the data stored in the IPO rendered at an overlooking viewpoint. Figure 21 shows a sequence of images synthesized by using the reconstructed textured-mapped 3-D meshes.

\section{CONCLUSION}

We have presented a new approach to reconstructing the 3-D environment automatically with an active binocular head. Active vision has been advocated by many researchers, for example, Bajcsy, Aloimonos, and Ahuja, about a decade ago. However, most active stereo vision systems have been applied to object tracking and not much progress on 3-D reconstruction using active stereo has ever been made after Ahuja and Abbott's work mainly because calibrating an active binocular head is much more difficult than calibrating a fixed camera. We have spent many years calibrating our binocular head and have achieved very accurate calibration results [12]. Based on our well-calibrated binocular head, we have developed an active stereo vision algorithm which can estimate the 3-D depth automatically, plan and maneuver a sequence of local movements to reduce the ambiguity in stereo matching, and integrate 3-D data obtained in different points of observation. Real experiments 
have been performed to verify the algorithm proposed in this paper. The experimental results show that the proposed algorithm is promising.

\section{APPENDIX}

\section{The Ray Tracing Method for Synthesizing an Image from the IPO}

Let us choose the reference frame of the IPO as the world coordinate system (WCS). Given a set of intrinsic and extrinsic camera parameters, we can back-project an image point, say $P_{2 D}$, into a 3-D ray. The back-projected 3-D ray is then transformed into the WCS and the resulting line equation is given by

$$
\left[\begin{array}{l}
x \\
y \\
z
\end{array}\right]=P_{0}+\lambda P_{1}
$$

where $P_{0}$ is the projection center of the camera measured in the WCS, $P_{1}$ is the direction of the 3-D ray, and $\lambda$ is a positive number. Converting the Cartesian coordinates $(x, y, z)$ into spherical coordinates, we have $(\rho, \theta, \phi)$, where

$$
\rho=\sqrt{\left\|P_{0}\right\|^{2}+2 \lambda P_{0}^{t} P_{1}+\lambda^{2}\left\|P_{1}\right\|^{2}}
$$

From the above equation, we can derive a representation of the positive number $\lambda$ as follows:

$$
\lambda=\frac{-2 P_{0}^{t} P_{1}+\sqrt{\left(P_{0}^{t} P_{1}\right)^{2}-4\left\|P_{1}\right\|^{2}\left(\left\|P_{0}\right\|^{2}-\rho^{2}\right)}}{2\left\|P_{1}\right\|^{2}} .
$$

Substituting (A.3) into (A.1), the 3-D line is now parameterized by $\rho$, namely $(x(\rho), y(\rho)$, $z(\rho))$. To search for a surface voxel by using the back-projected 3-D line, the 3-D line is mapped to a 3-D curve with parameter $\frac{1}{\rho}$ in the IPO. Given the the curve parameter $\frac{1}{\rho}$, we can compute the 3-D Cartesian coordinates of a point on the line $(x(\rho), y(\rho), z(\rho))$, from which we can determine $\theta$ and $\phi$. If $\left(\frac{1}{\rho}, \theta, \rho\right)$ is the first voxel intersecting the back-projected 3 -D line, then the color data contained in this voxel are used to fill the pixel, i.e., $P_{2 D}$, on the output image. For each pixel of the output image, the ray tracing procedure is proceeded to synthesize the output image.

\section{ACKNOWLEDGMENT}

This work was supported in part by National Science Council of Taiwan, ROC, under Grant NSC 86-2745-E001-002.

\section{REFERENCES}

1. U. R. Dhond and J. Aggarwal, Structure from stereo-A review, IEEE Trans. Systems, Man, Cybernet. 19(6), 1989, 1489-1510.

2. P. E. Debevec, C. J. Taylor, and J. Malik, Modeling and rendering architecture from photographs: A hybrid geometry- and image-based approach, in Proceedings of ACM SIGGRAPH'96, 1996, pp. 11-20. 
3. Y.-P. Hung, K.-C. Hung, C.-S. Chen, and C.-S. Fuh, Multi-pass hierarchical stereo matching for generation of digital terrain models from aerial images, Machine Vision Appl. 11(1), 1998, 280-291.

4. J. Aloimonos and A. Badyopadhyay, Active vision, in Proceesings of the First International Conference on Computer Vision, 1987, pp. 35-54.

5. J. Aloimonos, Purposive and qualitative active vision, in Proceedings of the International Conference on Pattern Recognition, 1990, Vol. 1, pp. 346-360.

6. I. D. Reid and P. A. Beardsley, Self-alignment of a binocular robot, Image Vision Comput. 14, 1996, 635-640.

7. P. F. McLauchlan and D. Murray, Active camera calibration for a head-eye platform using the variable statedimension filter, IEEE Trans. Pattern Anal. Mach. Intell. 18, 1996, 15-22.

8. M. Li, Camera Calibration of the kth Head/Eye System, Tech. Rep. CVAP147, Computational Vision and Active Perception Laboratory, Department of Numerical Analysis and Computing Science, Royal Institute of Technology (KTH), S-100 44, Stockman, Sweden, 1994.

9. M. Li and J. M. Lavest, Some Aspects of Zoom-Lens Camera Calibration, Tech. Rep. CVAP172, Computational Vision and Active Perception Laboratory, Department of Numerical Analysis and Computing Science, Royal Institute of Technology (KTH), S-100 44, Stockman, Sweden, 1995.

10. M. Li, D. Betsis, and J. M. Lavest, Kinematic Calibration of the kth Head-Eye System, Tech. Rep. CVAP171, Computational Vision and Active Perception Laboratory, Department of Numerical Analysis and Computing Science, Royal Institute of Technology (KTH), S-100 44, Stockman, Sweden, 1994.

11. M. Li and D. Betsis, Head-eye calibration, in Proceedings of the International Conference on Computer Vision, 1995, pp. 40-45.

12. S.-W. Shih, Y.-P. Hung, and W.-S. Lin, Calibration of an active binocular head, IEEE Trans. Systems Man Cybernet.-Part A: Systems Humans 28, 1998, 426-442.

13. K. Brunnstrom, J.-O. Eklundh, and T. Uhlin, Active fixation for scene exploration, Internat. J. Comput. Vision 17(2), 1996, 137-162.

14. H. Wu, T. Fukumoto, Q. Chen, and M. Yachida, Active face observation system, in Proceedings of the International Conference on Pattern Recognition, 1996, Vol. 3, pp. 441-445.

15. A. Davison and D. Murray, Mobile robot localisation using active vision, in Proceedings of the European Conference on Computer Vision, 1998, pp. 809-825.

16. I. D. Reid and D. W. Murray, Active tracking of foveated feature clusters using affine structure, Internat. J. Comput. Vision 18(1), 1996, 41-60.

17. M. Kam, X. Zhu, and P. Kalata, Sensor fusion for mobile robot navigation, in Proceedings of the IEEE, 1997, Vol. 85, pp. 108-119.

18. S. M. Seitz and C. R. Dyer, Photorealistic scene reconstruction by voxel coloring, in Proceedings of the IEEE Conference on Computer Vision and Pattern Recognition, 1997, pp. 1067-1073.

19. C.-Y. Lin, S.-W. Shih, and Y.-P. Hung, Toward automatic reconstruction of 3D environment with an active binocular head, in Proceedings of the International Conference on Pattern Recognition, 1998, Vol. 2, pp. 1708 1710.

20. K. N. Kutulakos and S. M. Seitz, A theory of shape by space carving, in Proceedings of the International Conference on Computer Vision, 1999, pp. 307-314.

21. R. Beß, D. Paulus, and H. Niemann, 3D recovery using calibrated active camera, in Proceedings of the IEEE International Conference on Image Processing, 1996, pp. 855-858.

22. N. Maru, A. Nishikawa, F. Miyazaki, and S. Arimoto, Active binocular stereo, in Proceedings of the IEEE Conference on Computer Vision and Pattern Recognition, 1993, pp. 724-725.

23. E. Grosso and M. Tistarelli, Active/dynamic stereo vision, IEEE Trans. Pattern Anal. Mach. Intell. 17, 1995, $868-879$.

24. N. Ahuja and L. Abbott, Active stereo: Integrating disparity, vergence, focus, aperture, and calibration for structure estimation, IEEE Trans. Pattern Anal. Mach. Intell. 15(10), 1993, 1007-1029.

25. E. Marchand and F. Chaumette, An autonomous active vision system for complete and accurate 3D scene reconstruction, Internat. J. Comput. Vision 32(3), 1999, 171-194.

26. Y.-P. Hung, D. B. Cooper, and B. Cernuschi-Frias, Asymptotic Bayesian surface estimation using an image sequence, Internat. J. Comput. Vision 6(2), 1991, 105-132. 
27. M. Okutomi and T. Kanade, A multiple-baseline stereo, IEEE Trans. Pattern Anal. Mach. Intell. 15(4), 1993, 353-363.

28. S. D. Blostein and T. S. Huang, Error analysis in stereo determination of 3-D point positions, IEEE Trans. Pattern Anal. Mach. Intell. 9(6), 1987, 752-765.

29. C.-Y. Lin, S.-W. Shih, Y.-P. Hung, and G. Y. Tang, Toward Automatic Reconstruction of 3D Environment with an Active Binocular Head, Tech. Rep. TR-IIS-01-009, Institute of Information Science, Academia Sinica, Taipei 115, Taiwan, 2001.

30. D. B. Cooper, J. Subrahmonia, Y.-P. Hung, and B. Cernuschi-Frias, The Use of Markov Random Fields in Estimating and Recognizing Objects in 3D Space Markov Random Fields: Theory and Applications (Rama Chellapa and Anil Jain, Eds.), Academic Press, San Diego, 1993. 